\title{
Once a Caesarean, Why a Caesarean?
}

\author{
Frances Handley-Derry
}

Almost everything that I have written in the first year of my training in a Masters Program has begun with "the rate of Caesarean section is rising in Canada", and yet again I start with the same reiteration. As this will eventually be the topic of my thesis, it is reasonable that it has become something I think about often. Currently, Caesarean sections (C-sections) represent approximately $26.3 \%$ of the births in Canada (1). There is concern among health care professionals over this rate due to the risks associated with $\mathrm{C}$-section including: complication with the anaesthesia, residual pain at the incision site affecting mother-child bonding, and potential complications in future pregnancies (2). So, if we know the risks, and experts think that there should be fewer performed, why are C-section rates still rising?

The main media coverage of high C-section rates narrows in on the so-called "too posh to push" phenomenon, (or should that be "too Posh to push"?) where celebrities such as Victoria Beckham, Madonna and Jessica Simpson book C-sections to avoid labour. Apart from other areas sporting large, wealthy communities, such as Chelsea in London, and Los Angeles, is this really representative of national trends? Since starting my master's I have some first-hand experience talking to women who have $\mathrm{C}$-sections and none that I have encountered so far fit into this box. Driving this trend is the experience of the average woman; therefore what we need to think about is how we have changed so that $\mathrm{C}$-sections are now a more common practice.

There are of course physical trends seen in Canada that fit with a higher C-section rate. Women are giving birth on average at a later age, potentially leading to more complications requiring a C-section. Having an increased percentage of the

Frances Handley-Derry

Masters Candidate, Department of Family Medicine McGill University female population being overweight, or obese, and the medical conditions associated with this, such as diabetes, may make physicians (or the women themselves) less likely to want to go through labour if the end results are the same as doing a C-section in the first place. However, these reasons can only account for a small part of the explanation.

Slowly and surely we have moved childbirth away from the 'natural' realm, the baby being delivered at home with the help of women in the community, to the 'medical realm', with the delivery in the hospital. Of course, this immediate access to medical technology has dramatically reduced the number of women and babies that die during childbirth by being able to provide interventions, such as C-sections. The other side of the 'medicalization' of birth is that women have become detached from the process. Many women do not know what to expect. They may experience fear about the labour, and a sense that they will not be able to have a successful vaginal delivery, particularly in the case of women who have previously had a Caesarean due to failure to progress in labour.

I would now like to focus on women who have repeat C-sections, which represents a significant part of the increasing rate. It used to be that once a woman had a C-section, she would be delivered this way for all subsequent pregnancies. Now, obstetrical recommendations are in favour of vaginal birth after caesarean (VBAC), but this does not seem to be translating into fewer caesarean births. In our culture, we have become very risk intolerant. Delivering vaginally after a caesarean is associated with the risk of uterine rupture; a very rare complication, but one that can be potentially fatal for the baby. When I talk to women about risks of VBAC, this is something that most women remember discussing.

One thing I find particularly interesting is that reasons for having a C-section are almost always framed in the negative for having a vaginal birth- something like "I have diabetes, so I can't 
delivery vaginally". What I think we need to be doing is increasing women's confidence in themselves, and in the natural process of childbirth if we want to see any change in the C-section rate. But the healthcare system has to change as well to support this. If we want to understand national trends, we should stop talking about the posh minority, and focus instead on examining how our everyday assumptions have changed to make the $\mathrm{C}$-section rate what it is today.

\section{REFERENCES}

1. Giving Birth in Canada: Regional Trends From 2001-2002 to 2005-2006. [Internet]. Ottawa: Canadian Institute for Health Information; 2007 [cited 2012 May 20]. Available from http://secure.cihi.ca/cihiweb/products/childbirth_ aib_070725_e.pdf

2. Dickerson, $\overline{\mathrm{T}}$. The Rise and Fall of VBAC in the United States. Journal of Legal Nurse Consulting. 2010; 21(1):3-8.

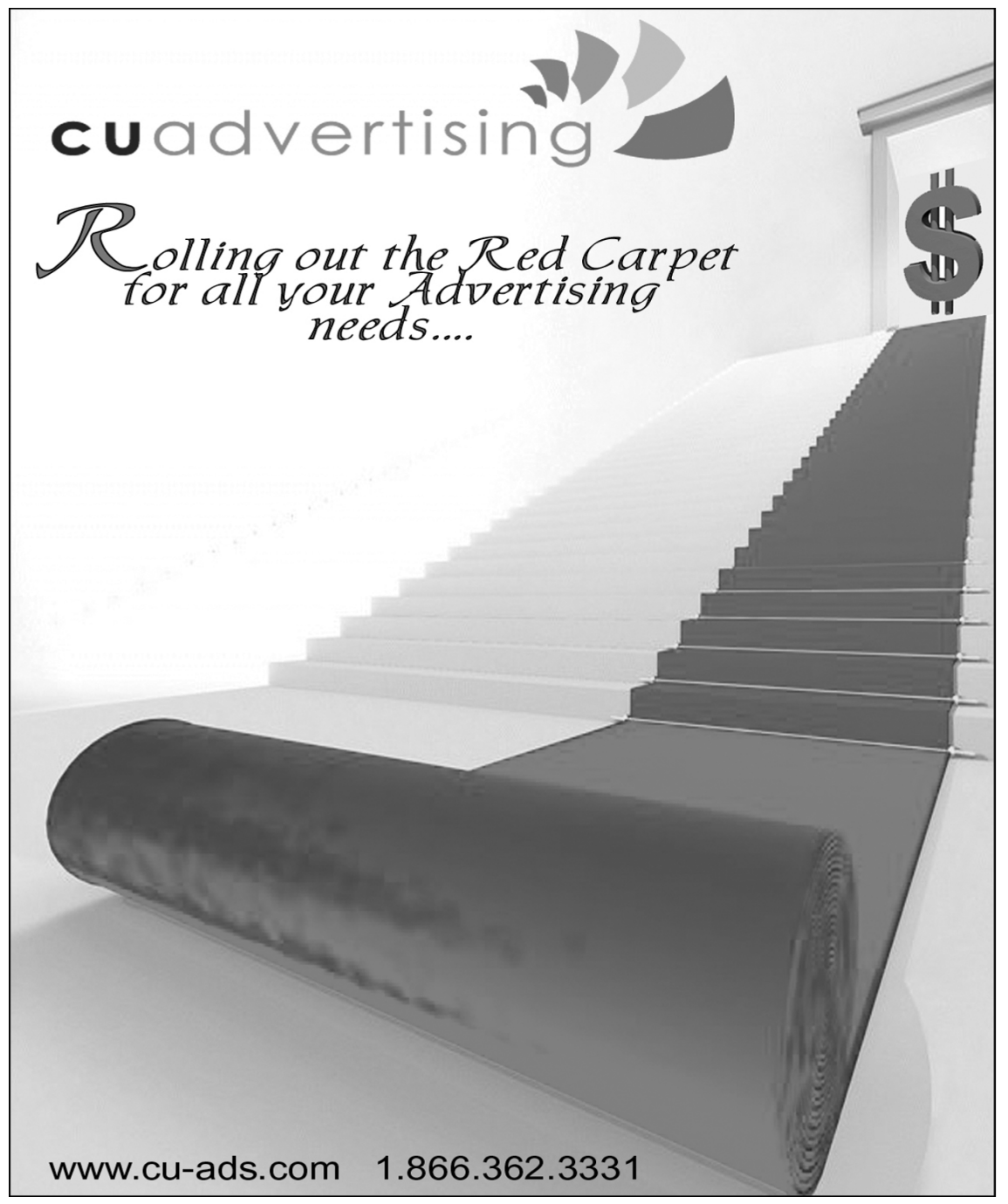

